\title{
Association of Soybean Food Intake and Cardiometabolic Syndrome in Korean Women: Korea National Health and Nutrition Examination Survey (2007 to 2011)
}

\author{
Sook-Hyun Jun ${ }^{1}$, Woo-Kyoung Shin ${ }^{2}$, Yookyung Kim ${ }^{1}$ \\ ${ }^{1}$ Department of Human Ecology, Graduate School of Korea University, Seoul, \\ ${ }^{2}$ Department of Preventive Medicine, Seoul National University College of Medicine, Seoul, Korea
}

\begin{abstract}
Background: Soybean food consumption has been considered as a possible way to lower incidence of cardiometabolic syndrome (CMS) among Asians. However, results from studies investigating its efficacy on CMS in Asians have been inconsistent.

Methods: We analyzed the association between soybean intake frequency and prevalence of CMS based on data from the Korea National Health and Nutrition Examination Survey 2007 to 2011. Data of 9,287 women aged 20 to 64 years were analyzed. Food frequency questionnaire was used to assess soybean food consumption frequency. General linear model and multivariable logistic regression model were used to examine the association of soybean intake quintile with CMS and its risk factors. Least square means of metabolic factors mostly showed no significant relevance except liver indexes.

Results: Compared to participants in the 1st quintile ( $<2$ times/week of soybean food), odds ratios (OR) for CMS and abdominal obesity (AO) in the 4 th quintile ( 8.5 times/week < soybean food $\leq 17$ times/week) were 0.73 (95\% confidence interval [CI], 0.57 to 0.95 ) and 0.72 (95\% CI, 0.58 to 0.90$)$, respectively. After excluding Tofu products, ORs of CMS, AO, high blood pressure, and hypertriglyceridemia were lower than those without excluding Tofu products. However, results still did not show significant inverse linear trend across frequency quintiles.

Conclusion: Our findings suggest that soybean intake of 8.5 to 17 times/week was inversely associated with CMS in Korean women. The relation between soybean intake $>17$ times/week and CMS varied depending on soybean food items.
\end{abstract}

Keywords: Diabetes mellitus; Korea; Obesity, abdominal; Soy foods

\section{INTRODUCTION}

Increasing prevalence of cardiometabolic syndrome (CMS) is a health concern worldwide. According to the World Health Organization, some developing countries with population adhering to their traditional eating habits such as those in South East Asia do not show increase in incidence of CMS. However, countries that adapted to western diet such as Iran had incidence of CMS that was even higher than certain developed countries [1]. Similarly, the prevalence of type 2 diabetes mellitus (T2DM), one of conditions of CMS, has been lower in Asian populations than that in Western countries [2]. CMS prevalence in South Korea increased between the mid-1990s and mid-2000s. However, in 2013, it was $28.9 \%$ without a signifi-
Corresponding authors: Yookyung Kim (D) https://orcid.org/0000-0002-8438-0121 Department of Human Ecology, Graduate School of Korea University, 145 Anam-ro, Seongbuk-gu, Seoul 02841, Korea

E-mail: yookyung_kim@korea.ac.kr
This is an Open Access article distributed under the terms of the Creative Commons Attribution Non-Commercial License (http://creativecommons.org/licenses/by-nc/4.0/) which permits unrestricted non-commercial use, distribution, and reproduction in any medium, provided the original work is properly cited.

Woo-Kyoung Shin (D) https://orcid.org/0000-0003-2725-4652

Department of Preventive Medicine, Seoul National University College of Medicine,

101 Daehak-ro, Jongno-gu, Seoul 03080, Korea

E-mail: shiningwk@gmail.com 
cant increasing or decreasing trend for the past 5 years [3]. On the contrary, in the United States, nearly $35 \%$ of all adults and $50 \%$ of those aged 60 years or older were estimated to have CMS in 2012. The rate had been increasing for the past 10 years [4].

Soybeans and other processed soybean food have been consumed for a long time in Asia to compensate incomplete protein content in rice. Asians typically consume 9 to $30 \mathrm{~g}$ of soybeans per day, with individual and regional variations [5]. According to the Korean National Nutrition Survey [6], daily mean intake of total genistein and daidzein in the Korean population is estimated to be $21.0 \mathrm{mg}$ per person. This means that a Korean consumes more isoflavones than a person who lives in United States or Europe [7]. Dietary isoflavone is consumed by only $35 \%$ of adults in a day with an average intake of $3.1 \mathrm{mg} /$ day, resulting in a mean intake of $1.0 \mathrm{mg} /$ day for all United States adults [8]. Frequent intake of cultivated soybean food which is unique to traditional Asian cuisines is not common in western diet. This seems to have relation with the lower incidence of CMS among Asians $[9,10]$.

Although there are several epidemiologic and experimental data reporting an inverse relationship of the consumption of soybean with several metabolic disorders [11-13], several studies have found no clear association between soy intake and incidence of cardio metabolic disorders [10,14,15]. According to some randomized clinical trials, soybean food consumption does not show any improvement for insulin resistance, serum glucose level, or lipid profiles $[16,17]$. Furthermore, soybean intake tends to have sex dependent effects on risk of CMS and specific cancer $[18,19]$.

Overall, research results about the effect of soybean consumption on CMS, especially among metabolically healthy women, were inconsistent $[10,14,20]$. Papers suggesting that soybean consumption was not associated with CMS were mostly based on people who did not routinely eat soybean food or eat small amounts of them $[17,21]$. Thus, it is necessary to examine effects of soybean foods on healthy women who eat soybeans on a daily basis.

For South Korean population, studies on this topic have also shown conflicting results recently. Several findings suggested that soybeans had protective effects against CMS among obese women $[22,23]$. However, other studies did not find a significant relation between soybean food intake and CMS [20]. Thus, the objective of this study was to investigate the association between CMS and soybean food intake among South Korean women.

\section{METHODS}

\section{Participants}

In this study, we used data from the Korea National Health and Nutrition Examination Survey (KNHANES) IV and V (2007 to 2011). It contains health interview survey, physical examination research, and nutrition questionnaires. Stratified multistage sampling design was used. Sampling was done according to geographical area, residential environmental type, age, and sex. All participants provided written informed consent before their participation. Initial candidates for the present study were those having completed nutrition questionnaires. We then excluded subjects who were men $(n=12,891)$ and those who were under 19 years of age or over 64 years of age $(n=5,194)$. Additionally, we excluded people who reported to intake implausible amount of total energy $(n=85 ;<450$ or $>6,300 \mathrm{kcal} /$ day for women) as data from those participants could not be applied to the general population. They could cause distortion of data. We excluded people whose daily energy intake was less than $25 \%$ or over $300 \%$ of the estimated energy requirements (EER). According to the Dietary Reference Intakes for Koreans 2015 (KDRI) [24], the EER for the adult is from 1,800 to $2,100 \mathrm{kcal}$, with less than $25 \%$ being 450 and over $300 \%$ being $6,300 \mathrm{kcal}$ [24,25]. Pregnant $(n=198)$ and lactating women $(n=282)$ were also excluded because their physiological conditions were changed during pregnancy and breast feeding. We also excluded those who had already been diagnosed by their doctors with diabetes mellitus $(n=429)$, hypertension $(n=1,330)$, or hyperlipidemia $(n=399)$. In addition, we excluded subjects who had not completed anthropometric examination or a blood test $(n=893)$. Finally, 9,287 participants were included in the final analysis. Fig. 1 shows the entire flow of participant selection.

\section{Demographic and health behavior}

Trained interviewers collected data of demographic factors and health behaviors of participants via personal interviews. Demographic variables included age, sex, achieved educational level (high school education or less, and college education or more), and monthly household income (tertile of equivalized household income). Equivalized household income was calculated as total monthly household income divided by the square root of the total number of household members. Health behavioral variables included smoking, alcohol consumption, and physical activity. Participants were asked to choose whether they were never smokers or were past or present smokers. The 


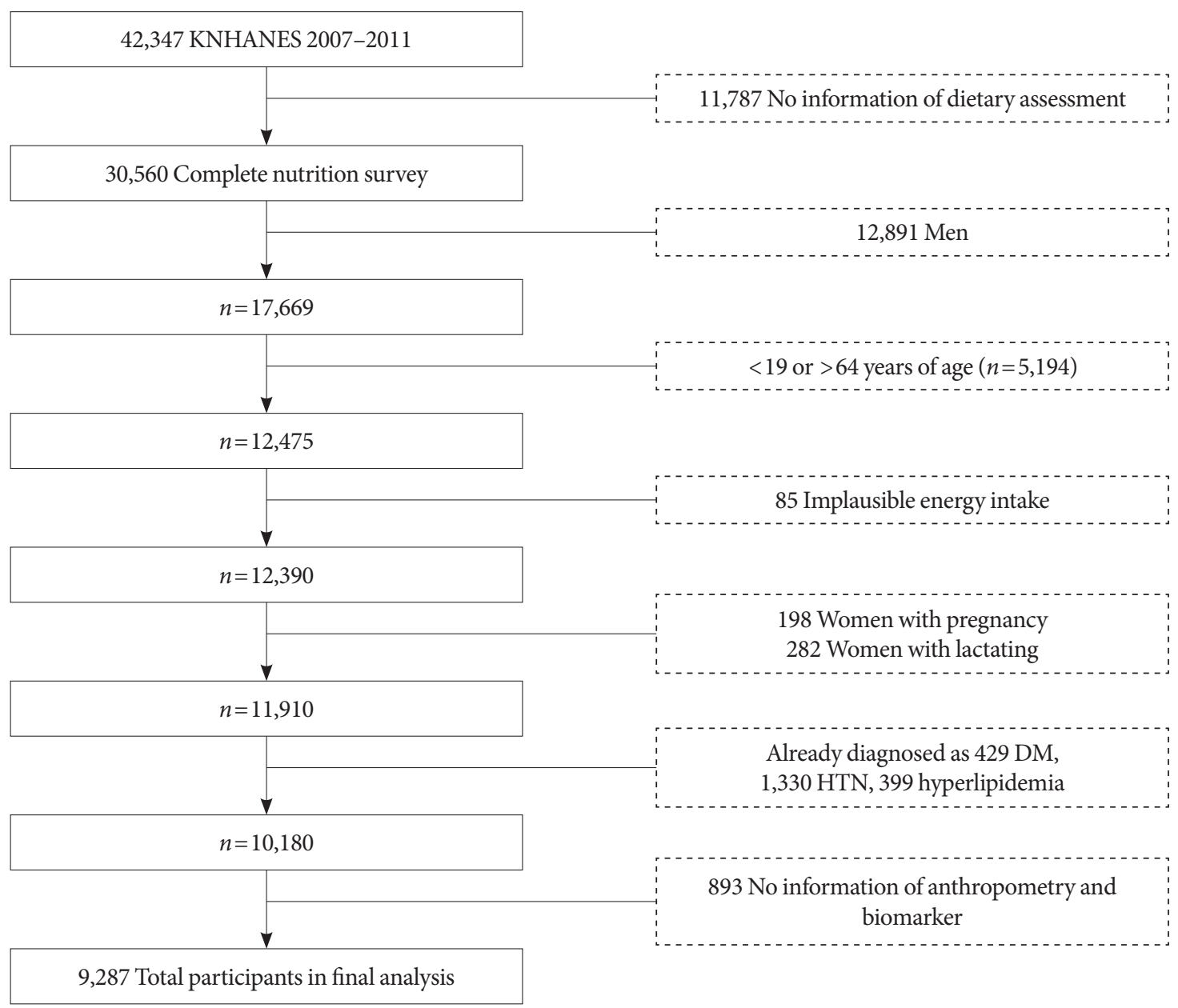

Fig. 1. Flow chart of participants. KNHANES, Korea National Health and Nutrition Examination Survey; DM, diabetes mellitus; HTN, hypertension.

average amount and number of consumed alcoholic beverages was assessed by self-questionnaire. It was then assigned into five groups based on frequency per week. Physical activity was quantified as metabolic equivalent of task minutes per 7 days (MET-minutes per week). It was calculated using the scoring protocol of the Korean version of the International Physical Activity Questionnaire (IPAQ) form [26]. MET means energy expenditure per $\mathrm{kg} /$ minute $(\mathrm{kcal} / \mathrm{min} / \mathrm{kg})$. MET-minutes was calculated as 'MET level of each behavior $\times$ minute $\times$ frequency/ week.' Met level of light intensity activities (walking $4.8 \mathrm{~km} / \mathrm{hr}$ ) was 3.3 METs. Moderate intensity activities had 4.0 METs and vigorous intensity activities had 8.0 METs. Participants were asked to choose whether they performed low, moderate, or high physical activity. Physical activity levels were then classified as low (<600 MET-minutes per week), moderate (more than 600 but less than 3,000 MET-minutes per week), or high
(>3,000 MET-minutes per week).

\section{Dietary assessment}

Dietary assessments were conducted by using food frequency questionnaire (FFQ). FFQ consisted of 63 mainly consumed food items in South Korea with 10 categories of frequency value (almost never, 6 to 11 times/year, 1 time/month, 2 to 3 times/ month, 1 time/week, 2 to 3 times/week, 4 to 6 times/week, 1 time/day, 2 times/day, 3 times/day). Soybean food consisted of three items: soybean group (which contained rice with soybean, beans cooked in soy sauce, etc.), tofu group (which contained tofu put in soup, stew, flat cake, boiled down in soy sauce or other seasonings, soft tofu), and soybean milk group. All analyses accounted for the complex sampling design effect and appropriate sampling weights of the national survey. We converted the soybean FFQ into daily intake frequency and 
then grouped it into quintile according to the number of participants proportionally. Energy intake variables used in this study were extracted from 24-hour recall data. They included total energy (kcal/day), carbohydrate (g/day), protein (g/day), total fat (g/day), and sodium (mg/day) intakes presented as total energy adjusted values using the residual method.

\section{Anthropometric examination and blood test}

Physical examination was performed by well-trained medical staff following standard procedures. Body weight and height were measured to $0.1 \mathrm{~kg}$ and $0.1 \mathrm{~cm}$, respectively, with participant wearing indoor clothing without shoes. Waist circumference (WC) was measured at the narrowest point between the lower border of the rib cage and the iliac crest. Body mass in$\operatorname{dex}(\mathrm{BMI})$ was calculated as the ratio of weight $(\mathrm{kg}) /$ height squared $\left(\mathrm{m}^{2}\right)$. Blood pressure (BP) was measured for the right arm using a mercury sphygmomanometer (Baumanometer 0850 wall unit 33; W.A. Baum Co. Inc., Copiague, NY, USA) three times per subject by trained nurses. Before 2011, the height of the arm on which BP was measured was different by year. Thus, we used corrected BP variables to prevent the issue of combining BP data from different years. Levels for total cholesterol (TC), high density lipoprotein cholesterol (HDL-C), triglyceride (TG), and glucose, and so on were measured using blood samples obtained from the antecubital vein after a 12hour overnight fast. Fasting plasma glucose (FPG), TC, TG, and HDL-C levels were evaluated using a Hitachi 700-110 Chemistry Analyzer (Hitachi, Tokyo, Japan). Fasting insulin levels were estimated by immunoradiometric assay (Biosource, Louvain-la-Neuve, Belgium) using a $\gamma$-counter (1470 Wizard; PerikinElmer, Turku, Finland). glycosylated hemoglobin (HbAlc) was measured by high performance liquid chromatography (HLC-723G7; Tosch, Tokyo, Japan).

\section{Definition of cardiometabolic syndrome and cardiometabolic syndrome score}

CMS is a combination of metabolic disorders or risk factors, including a combination of diabetes mellitus, systemic arterial high BP, central obesity, and hyperlipidemia. Definitions for CMS and its components were obtained from the National Cholesterol Education Program Adult Treatment Panel III guideline. We used the ethnicity-specific values for WC based on data from the World Health Organization and the Korean Society for the Study of Obesity [27]. CMS was defined by the presence of three or more of the following risk factors: central obesity
(WC $\geq 90 \mathrm{~cm}$ for men, and $\geq 85 \mathrm{~cm}$ for women); high BP (systolic $\mathrm{BP} \geq 130 \mathrm{~mm} \mathrm{Hg}$ and diastolic $\mathrm{BP} \geq 85 \mathrm{~mm} \mathrm{Hg}$, or using antihypertension drug); fasting glucose levels $\geq 100 \mathrm{mg} / \mathrm{dL}$; TG levels $\geq 150 \mathrm{mg} / \mathrm{dL}$; and low HDL-C levels ( $<40 \mathrm{mg} / \mathrm{dL}$ for men, and $<50 \mathrm{mg} / \mathrm{dL}$ for women). If each criterion is met, a score of one point is imposed. We named this as CMS score, ranging from 0 to 5 points. Those with CMS score of more than 3 points were classified as having metabolic syndrome (MetS).

\section{Definitions of other cardio metabolic variables}

Homeostasis model assessment (HOMA) is the most broadly used in epidemiologic research [28]. Homeostasis model assessment of insulin resistance (HOMA-IR) is a test for insulin resistance while HOMA- $\beta$ indicates insulin formation potential regarding pancreatic $\beta$-cell function [28]. These two HOMA parameters are calculated as follows:

$$
\text { HOMA-IR=fasting insulin }(\mu \mathrm{IU} / \mathrm{mL}) \times \mathrm{FPG}(\mathrm{mg} / \mathrm{dL}) /
$$$$
22.5 \times 18
$$

$$
\text { HOMA- } \beta=20 \times \text { fasting insulin }(\mu \mathrm{IU} / \mathrm{mL}) / \mathrm{FPG}(\mathrm{mg} / \mathrm{dL}) /
$$

18-3.5

Nonalcoholic fatty liver disease (NAFLD) is defined as the presence of cytoplasmic lipid droplets in more than $5 \%$ of hepatocytes in individuals without significant alcohol consumption and negative viral and autoimmune liver diseases [29]. Presence of NAFLD has recently been considered as the hepatic component of CMS as it is a result of obesity for which there is ectopic accumulation of TG in the liver parenchyma [30]. Based on significant evidence, NAFLD appears to play an important role in the disease mechanism of metabolic disorders [30].

As a standard diagnosis for NAFLD, "NAFLD liver fat score" is mainly based on magnetic resonance spectroscopy [31]. It has $95 \%$ sensitivity and specificity.

NAFLD liver fat score $=-2.89+1.18 \times($ CMS: yes $=1 /$ no $=0)+$ $0.45 \times(\mathrm{T} 2 \mathrm{DM}$ : yes $=1 / \mathrm{no}=0)+0.15 \times($ fasting serum insulin, $\mu \mathrm{IU} / \mathrm{mL})+0.04 \times($ AST, IU/L) $-0.94 \times($ AST/ALT $)$

\section{Statistical analysis}

All statistical analyses were performed using SAS software version 9.4 (SAS Institute Inc., Cary, NC, USA). All analyses accounted for the complex sampling design effect and appropriate sampling weights of the national survey using SAS Proc survey. We applied a sample weighting in the analysis using weight code in Proc survey procedure. A two-tailed $P$ value 
$<0.05$ was considered to be statistically significant. Values for demographic, health-related behavior, and biochemical variables were expressed as mean \pm standard deviation (SD) for continuous variables or number of participants (percentage) for categorical variables. Calculated probability derived from either analysis of variance (ANOVA) comparing mean values (for continuous variables) or chi-square tests comparing distribution of categorical variables was used to test differences by soybean food intake.

Cardio metabolic factors derived from several blood test results are expressed as mean $\pm \mathrm{SD}$ across quintiles of soybean food intake. Significance determined by general linear model (GLM) with Tukey multiple comparisons test $(P<0.05)$ after adjusting for education (high school or less, college or more), income (tertile of equivalized household income), physical activity (low, moderate, high), smoking status (nonsmoker, exsmoker, current smoker), alcohol consumption (times/week), total energy (kcal/day), carbohydrate (g/day), total fat (g/day), protein (g/day), and sodium ( $\mathrm{mg} / 1,000 \mathrm{kcal})$ intakes as continuous variables. We used the residual method to account for effects of total energy intake on each nutrient as a confounding factor to CMS. PROC GLM procedure was used to examine a linear trend ( $P$ for trend) across soybean consumption categories by using median value within each exposure category.

Multivariable logistic regression (MLR) analysis was performed to estimate the odds ratio (OR) and 95\% confidence interval (CI) of individual component of CMS according to soybean food intake quintiles using the lowest quintile as reference. We developed three different models which adjusted confounders. When we chose covariates for multivariable model, we referred to results of analysis for baseline characteristics of study subjects by soybean food consumption. ORs were initially calculated following adjustment for age in model 1. In model 2, categorical variables were further adjusted for education, monthly household income, physical activity, smoking status, and alcohol consumption activity. Dietary factors such as total energy, carbohydrate, protein, total fat, and sodium intakes were additionally adjusted for as continuous variables in model 3. We applied three types of model to know the influence of each model of confounders on ORs. The prevalence of high BP, hyperlipidemia, T2DM, myocardial infarction/ angina pectoris, stroke was compared across soybean food intake groups. MLR analyses were performed to estimate ORs and 95\% CI with the lowest quintile group as the reference group after adjusting for confounding variables by the three models as described above.

\section{RESULTS}

\section{General characteristics of participants according to soybean food intake}

According to soybean food consumption, CMS score, socio demographic, anthropometric, and biochemical characteristics of participants are presented in Table 1. CMS score means the score corresponding to CMS component. With higher quintiles of soybean intake, age and high intensity exercise of participants also increased. The percentage of current smoker decreased with higher quintile of soybean intake. Statistical analysis results from either ANOVA comparing mean values or chi-square test comparing distribution of categorical variables showed significant difference by soybean food intake frequency. Variables such as age, CMS score, household income, education, smoking status, current alcohol intake, vigorous physical activity, and daily dietary intake were designated as confounders.

\section{Cardiometabolic factors according to soybean food intake}

Table 2 shows relationships between several variables related to cardio metabolic diseases and soybean product intake. When comparing average values, all variables were significantly $(P<$ 0.05) different across soybean intake quintiles except fasting serum insulin and HOMA-IR. Especially, the 5th quintile had significant difference in all variables except fasting serum insulin and HOMA-IR. However, the least square means of those variables after adjusting for various covariates (age, education, income, physical activity, smoking status, alcohol consumption, total energy intake, energy adjusted carbohydrate daily intake, energy adjusted protein daily intake, energy adjusted fat daily intake, energy adjusted sodium daily intake) significance disappeared except for aspartate aminotransferase/alanine aminotransferase (AST/ALT) and HOMA- $\beta$. $P$ values for trend in AST/ALT using median value of soybean product intake quintile after adjusting for the same confounders listed above showed marginally significant linearity across soybean food intake groups.

\section{Soybean food intake and cardiometabolic syndrome}

Soybean food intake was related to CMS and abdominal obesity $(\mathrm{AO})$ after adjusting for various confounders as shown in Table 3. Participants in the 4th (more than 8.5 to 17 or 17 times/ 
Table 1. General characteristics of participants according to soybean product intake

\begin{tabular}{|c|c|c|c|c|c|c|}
\hline \multirow{2}{*}{ Characteristic } & \multicolumn{6}{|c|}{ Soybean product intake } \\
\hline & Q1 & Q2 & Q3 & Q4 & Q5 & $P$ value $^{\mathrm{a}}$ \\
\hline Number & 1,845 & 1,861 & 1,969 & 1,794 & 1,818 & $<0.01$ \\
\hline $\begin{array}{l}\text { Soybean product intake frequency, } \\
\text { times/wk }\end{array}$ & $<2^{\mathrm{e}}$ & $\geq 2-\leq 4^{\mathrm{d}}$ & $>4-\leq 8.5^{c}$ & $>8.5-\leq 17^{b}$ & $>17^{\mathrm{a}}$ & $<0.01$ \\
\hline Age, yr & $39.85 \pm 12.08^{\mathrm{cd}}$ & $39.29 \pm 10.40^{\mathrm{d}}$ & $40.07 \pm 10.85^{c}$ & $41.56 \pm 11.31^{\mathrm{b}}$ & $45.72 \pm 10.88^{\mathrm{a}}$ & $<0.01$ \\
\hline $\mathrm{BMI}, \mathrm{kg} / \mathrm{m}^{2}$ & $22.73 \pm 3.51^{\mathrm{b}}$ & $22.86 \pm 3.40^{\mathrm{b}}$ & $22.72 \pm 3.24^{\mathrm{b}}$ & $22.66 \pm 3.12^{\mathrm{b}}$ & $23.10 \pm 3.23^{\mathrm{a}}$ & $<0.01$ \\
\hline Waist circumference, $\mathrm{cm}$ & $76.63 \pm 9.79^{\mathrm{b}}$ & $76.44 \pm 9.24^{\mathrm{b}, \mathrm{c}}$ & $76.03 \pm 8.88^{\mathrm{b}, \mathrm{c}}$ & $75.91 \pm 8.83^{\mathrm{c}}$ & $77.53 \pm 8.96^{\mathrm{a}}$ & $<0.01$ \\
\hline \multicolumn{7}{|l|}{ Household income } \\
\hline Low & $240(13.00)$ & $198(10.63)$ & $152(7.71)$ & $139(7.74)$ & $181(9.95)$ & \\
\hline Middle & $1,121(60.75)$ & $1,072(57.60)$ & $1,148(58.30)$ & $971(54.12)$ & $1,036(56.98)$ & $<0.01$ \\
\hline High & $484(26.23)$ & $591(31.75)$ & $669(33.97)$ & $684(38.12)$ & $601(33.05)$ & \\
\hline \multicolumn{7}{|l|}{ Education } \\
\hline$\leq$ High school & $1,316(71.32)$ & $1,159(62.27)$ & $1,243(63.12)$ & $1,167(65.05)$ & $1,317(72.44)$ & \\
\hline$\geq$ College & $529(28.67)$ & $702(37.72)$ & $726(36.87)$ & $627(34.94)$ & $501(27.55)$ & $<0.01$ \\
\hline \multicolumn{7}{|l|}{ Smoking status } \\
\hline Non-smoker & $1,560(84.55)$ & $1,623(87.21)$ & $1,748(88.77)$ & $1,606(89.52)$ & $1,693(93.12)$ & \\
\hline Ex-smoker & $123(6.66)$ & $124(6.66)$ & $117(5.94)$ & $97(5.40)$ & $66(3.63)$ & $<0.01$ \\
\hline Current smoker & $162(8.78)$ & $114(6.12)$ & $104(5.28)$ & $91(5.07)$ & $59(3.24)$ & \\
\hline \multicolumn{7}{|l|}{ Current alcohol intake, time/wk } \\
\hline$<0.25$ & $961(52.08)$ & $927(49.81)$ & $1,016(51.59)$ & $997(55.57)$ & $1,119(61.55)$ & \\
\hline 0.25 & $259(14.03)$ & $284(15.26)$ & $255(12.95)$ & $247(13.76)$ & $258(14.19)$ & $<0.01$ \\
\hline $0.5-1$ & $417(22.60)$ & $440(23.64)$ & $477(24.22)$ & $382(21.29)$ & $320(17.60)$ & \\
\hline $2-3$ & $167(9.05)$ & $173(9.29)$ & $176(8.93)$ & $160(8.91)$ & $101(5.55)$ & \\
\hline$\geq 4$ & $41(2.22)$ & $37(1.98)$ & $45(2.28)$ & $28(1.56)$ & $20(1.10)$ & \\
\hline \multicolumn{7}{|l|}{ Vigorous physical activity (METS) $^{\mathrm{b}}$} \\
\hline Low & $1,108(60.05)$ & $1,071(57.54)$ & $1,055(53.58)$ & $918(51.17)$ & $931(51.21)$ & \\
\hline Moderate & $515(27.91)$ & $576(30.95)$ & $683(34.68)$ & $620(34.55)$ & $630(34.65)$ & $<0.01$ \\
\hline High & $222(12.03)$ & $214(11.49)$ & $231(11.73)$ & $256(14.26)$ & $257(14.13)$ & \\
\hline \multicolumn{7}{|l|}{ Daily dietary intakes ${ }^{c}$} \\
\hline Total energy, kcal/day & $1,661.36 \pm 618.44^{c}$ & $1,715.90 \pm 631.09^{\mathrm{a}, \mathrm{b}}$ & $1,726.30 \pm 642.96^{\mathrm{a}}$ & $1,681.20 \pm 643.53^{b, c}$ & $1,738.59 \pm 614.51^{\mathrm{a}}$ & $<0.01$ \\
\hline Carbohydrate $^{\mathrm{d}}, \mathrm{g} /$ day & $284.57 \pm 53.13^{\mathrm{b}}$ & $286.74 \pm 47.94^{\mathrm{b}}$ & $284.03 \pm 49.96^{\mathrm{b}}$ & $284.26 \pm 49.03^{\mathrm{b}}$ & $297.31 \pm 46.62^{\mathrm{a}}$ & $<0.01$ \\
\hline Protein $^{\mathrm{d}}, \mathrm{g} /$ day & $59.37 \pm 17.61^{\mathrm{d}}$ & $60.65 \pm 15.94^{c}$ & $61.63 \pm 17.31^{\mathrm{b}, \mathrm{c}}$ & $62.89 \pm 16.75^{\mathrm{a}}$ & $62.55 \pm 16.51^{\mathrm{a}, \mathrm{b}}$ & $<0.01$ \\
\hline Fat $^{\mathrm{d}}, \mathrm{g} /$ day & $34.46 \pm 17.49^{\mathrm{b}}$ & $34.48 \pm 15.86^{\mathrm{b}}$ & $35.59 \pm 16.05^{\mathrm{a}}$ & $35.72 \pm 16.29^{\mathrm{a}}$ & $31.62 \pm 14.90^{c}$ & $<0.01$ \\
\hline Sodium ${ }^{\mathrm{d}}, \mathrm{mg} /$ day & $4,258.25 \pm 2,463.83^{\mathrm{b}}$ & $4,436.84 \pm 2,259.69^{\mathrm{a}}$ & $4,330.54 \pm 2,192.61^{\mathrm{a}, \mathrm{b}}$ & $4,321.68 \pm 2,313.47^{\mathrm{a}, \mathrm{b}}$ & $4,484.15 \pm 2,434.36^{\mathrm{a}}$ & 0.02 \\
\hline Cardiometabolic syndrome score ${ }^{e}$ & & & & & & 0.02 \\
\hline$<3$ & $1,609(87.21)$ & $1,656(88.98)$ & $1,764(89.58)$ & $1,612(89.85)$ & $1,563(85.97)$ & \\
\hline 3 & $167(9.05)$ & $144(7.73)$ & $137(6.95)$ & $134(7.46)$ & $191(10.50)$ & \\
\hline 4 & $53(2.87)$ & $49(2.63)$ & $59(2.99)$ & $41(2.28)$ & $55(3.02)$ & \\
\hline 5 & $16(0.87)$ & $12(0.64)$ & $9(0.45)$ & $7(0.39)$ & $9(0.49)$ & \\
\hline
\end{tabular}

Values are presented as mean \pm standard deviation or number $(\%)$.

METS, metabolic equivalent of task minutes.

${ }^{\mathrm{a}} P$ value derived from either analysis of variance (ANOVA), comparing mean values, or chi-square test comparing distribution of categorical variables, ${ }^{\mathrm{b}} \mathrm{Vig}$ (gorous physical activity (METS): low ( $<600$ MET-minutes per week), moderate ( $\geq 600$ to $<3,000$ MET-minutes per week), high ( $\geq 3,000$ MET-minutes per week), ${ }^{\circ}$ Dietary intake variables were obtained from 24-hour recall data of Korea National Health and Nutrition Examination Survey, ${ }^{\mathrm{d} D i e t a r y}$ intake variables, except for total energy, were energy adjusted using the residual method, ${ }^{\mathrm{e}} \mathrm{Cardiometabolic} \mathrm{syndrome} \mathrm{score:} \mathrm{cardiometabolic} \mathrm{syndrome} \mathrm{was} \mathrm{defined} \mathrm{using} \mathrm{the} \mathrm{National} \mathrm{Cho-}$ lesterol Education Program Adult Treatment Panel III criteria with a modified waist circumference cutoff for Korean adults if any three or more of the five components were present. Five components: (1) abdominal obesity (waist circumference $\geq 90 \mathrm{~cm}$ in male, $\geq 85 \mathrm{~cm}$ in female); (2) high blood pressure ( $\geq 130 / 85 \mathrm{~mm}$ $\mathrm{Hg}$ ); (3) fasting hyperglycemia ( $\geq 100 \mathrm{mg} / \mathrm{dL}$ ); (4) hypertriglyceridemia ( $\geq 150 \mathrm{mg} / \mathrm{dL})$; and (5) low HDL-C ( $<40 \mathrm{mg} / \mathrm{dL}$ in male, $<50 \mathrm{mg} / \mathrm{dL}$ in female). The number corresponding to the component was scored. 
Table 2. Cardiometabolic factors according to soybean product intake

\begin{tabular}{|c|c|c|c|c|c|c|c|}
\hline \multirow{2}{*}{ Variable } & \multicolumn{7}{|c|}{ Soybean product intake } \\
\hline & Q1 & Q2 & Q3 & $\mathrm{Q} 4$ & Q5 & $P$ value $^{\mathrm{a}}$ & $P$ for trend ${ }^{\mathrm{b}}$ \\
\hline \multicolumn{8}{|l|}{ Lipid metabolism } \\
\hline $\mathrm{TC}, \mathrm{mg} / \mathrm{dL}$ & $182.82 \pm 34.38$ & $182.52 \pm 33.68$ & $182.65 \pm 33.60$ & $184.62 \pm 33.31$ & $189.01 \pm 36.08$ & 0.77 & 0.38 \\
\hline HDL-C, mg/dL & $50.80 \pm 10.81$ & $50.98 \pm 10.69$ & $51.53 \pm 10.89$ & $51.64 \pm 11.20$ & $50.36 \pm 11.23$ & 0.23 & 0.25 \\
\hline LDL-C, mg/dL & $107.85 \pm 14.23$ & $107.98 \pm 13.97$ & $107.48 \pm 12.96$ & $107.66 \pm 14.17$ & $109.01 \pm 14.01$ & 0.32 & 0.32 \\
\hline $\mathrm{TG}, \mathrm{mg} / \mathrm{dL}$ & $101.92 \pm 72.47$ & $98.21 \pm 63.75$ & $97.09 \pm 67.42$ & $98.57 \pm 63.82$ & $106.14 \pm 68.36$ & 0.69 & 0.97 \\
\hline \multicolumn{8}{|l|}{ Blood pressure } \\
\hline SBP, mm Hg & $109.52 \pm 14.60$ & $108.48 \pm 13.72$ & $108.69 \pm 13.82$ & $108.89 \pm 13.53$ & $111.39 \pm 15.39$ & 0.26 & 0.10 \\
\hline DBP, mm Hg & $71.47 \pm 9.65$ & $71.27 \pm 9.40$ & $71.50 \pm 9.59$ & $71.68 \pm 9.12$ & $72.84 \pm 9.77$ & 0.96 & 0.85 \\
\hline \multicolumn{8}{|l|}{ Liver somatic value } \\
\hline AST, IU/L & $19.16 \pm 8.93$ & $19.02 \pm 8.16$ & $19.07 \pm 7.05$ & $19.54 \pm 12.20$ & $20.44 \pm 7.78$ & 0.72 & 0.18 \\
\hline ALT, IU/L & $16.56 \pm 14.57$ & $16.79 \pm 14.85$ & $16.44 \pm 11.61$ & $16.59 \pm 14.41$ & $18.20 \pm 11.04$ & 0.42 & 0.54 \\
\hline AST/ALT & $1.32 \pm 0.42$ & $1.31 \pm 0.41$ & $1.31 \pm 0.41$ & $1.32 \pm 0.88$ & $1.25 \pm 0.39$ & 0.03 & 0.07 \\
\hline NAFLD liver fat score & $-1.75 \pm 1.10$ & $-1.75 \pm 1.14$ & $-1.77 \pm 1.32$ & $-1.78 \pm 1.29$ & $-1.66 \pm 1.08$ & 0.63 & 0.52 \\
\hline \multicolumn{8}{|l|}{ Serum glucose/insulin level } \\
\hline FPG, mg/dL & $91.13 \pm 11.87$ & $91.13 \pm 11.57$ & $90.64 \pm 11.48$ & $90.99 \pm 12.42$ & $92.19 \pm 15.45$ & 0.58 & 0.64 \\
\hline Fasting serum insulin, $\mu \mathrm{IU} / \mathrm{mL}$ & $9.73 \pm 3.48$ & $9.80 \pm 4.19$ & $9.78 \pm 5.80$ & $9.72 \pm 4.32$ & $9.51 \pm 4.00$ & 0.53 & 0.36 \\
\hline HOMA-IR & $2.20 \pm 0.92$ & $2.23 \pm 1.13$ & $2.21 \pm 1.59$ & $2.20 \pm 1.18$ & $2.19 \pm 1.13$ & 0.61 & 0.44 \\
\hline HOMA- $\beta$ & $136.04 \pm 57.93$ & $138.08 \pm 99.56$ & $139.92 \pm 80.91$ & $138.12 \pm 101.42$ & $129.67 \pm 60.30$ & 0.24 & 0.53 \\
\hline $\mathrm{HbAlc}, \%$ & $5.57 \pm 0.32$ & $5.57 \pm 0.28$ & $5.55 \pm 0.26$ & $5.57 \pm 0.31$ & $5.60 \pm 0.36$ & 0.62 & 0.18 \\
\hline
\end{tabular}

Values are presented as mean \pm standard deviation.

TC, total cholesterol; HDL-C, high density lipoprotein cholesterol; LDL-C, low density lipoprotein cholesterol; TG, triglyceride; SBP, systolic blood pressure; DBP, diastolic blood pressure; AST, aspartate aminostransferase; ALT, alanine aminotransferase; NAFLD, nonalcoholic fatty liver disease; FPG, fasting plasma glucose; HOMA-IR, homeostatic model assessment of insulin resistance; HOMA- $\beta$, homeostatic model assessment of beta cell function; HbAlc, glycosylated hemoglobin.

a Significance determined by general linear model with Tukey multiple comparisons test $(P<0.05)$ after adjustments for age (continuous), education (high school or less, college or more), income (quartile of equivalized household income), physical activity (low, moderate, high), smoking status (never smoker, past smoker, current smoker), alcohol consumption (g/day quartile), total energy (kcal/day), carbohydrate (g/day), total fat (g/day), protein (g/day), sodium (mg/1,000 kcal) intakes as continuous variables. Sharing the same alphabet indicates no significant difference between two groups, ${ }^{\mathrm{b}} \mathrm{P}$ values for trend using median value of soybean product intake quintile after adjustments for confounders as same as above.

week) and the 5th (more than 17 times/week) quintiles of soybean food intake had significantly lower ORs for having CMS than those in the 1st quintile (less than 2 times/week) after adjusting for age ([OR, $0.63 ; 95 \% \mathrm{CI}, 0.49$ to 0.82 ] and [OR, 0.76; $95 \%$ CI, 0.60 to 0.97$]$, respectively). However, only ORs of the 4th quintile had significance after additionally adjusting for education, income, physical activity, smoking status, and alcohol consumption (in model 2), total energy intake, the three nutrient, and sodium intake (in model 3) ([OR, $0.71 ; 95 \% \mathrm{CI}, 0.55$ to 0.92 ] and [OR, $0.73 ; 95 \% \mathrm{CI}, 0.57$ to 0.95 ], respectively).

The 4th quintile of soybean food intake had significantly lower ORs of having AO than that the 1st quintile in models 1 , 2 , and 3 . The 5 th quintile of soybean food intake had $25 \%$ lower OR of having AO than the 1st quintile. However, after adjusting for various confounders in models 2 and 3 , significance attenuated. ORs for $\mathrm{AO}$ significantly decreased by the quintile of soybean food intake in model 3 ( $P$ for trend $<0.05$ ).

\section{Sensitivity analysis (soybean food intake and cardiometabolic syndrome)}

After excluding soybean or soybean milk items from FFQ data, there were no significant relevance of CMS or CMS compo- 
Table 3. Multivariable adjusted odds ratio for the cardiometabolic syndrome and its individual components

\begin{tabular}{|c|c|c|c|c|c|c|}
\hline \multirow{2}{*}{ Variable } & \multicolumn{6}{|c|}{ Soybean food intake } \\
\hline & Q1 & Q2 & Q3 & Q4 & Q5 & $P$ for trend \\
\hline \multicolumn{7}{|c|}{ Cardiometabolic syndrome $\mathrm{a}^{\mathrm{a}}$} \\
\hline No. of case (\%) & $236(12.7)$ & $205(11.0)$ & $205(10.4)$ & $182(10.1)$ & $255(14.0)$ & \\
\hline Model $1^{\mathrm{b}}$ & 1 & $0.87(0.67-1.12)$ & $0.79(0.61-1.01)$ & $0.63(0.49-0.82)$ & $0.76(0.60-0.97)$ & \\
\hline Model $2^{c}$ & 1 & $0.92(0.71-1.19)$ & $0.86(0.67-1.11)$ & $0.71(0.55-0.92)$ & $0.84(0.66-1.08)$ & \\
\hline Model $3^{\mathrm{d}}$ & 1 & $0.93(0.71-1.21)$ & $0.89(0.69-1.14)$ & $0.73(0.57-0.95)$ & $0.87(0.68-1.12)$ & 0.32 \\
\hline \multicolumn{7}{|c|}{ Abdominal obesity ${ }^{\mathrm{a}}$} \\
\hline No. of case (\%) & $361(19.5)$ & $316(16.9)$ & $314(15.9)$ & $272(15.1)$ & $358(19.6)$ & \\
\hline Model 1 & 1 & $0.88(0.71-1.08)$ & $0.81(0.65-1.00)$ & $0.64(0.52-0.79)$ & $0.75(0.61-0.92)$ & \\
\hline Model 2 & 1 & $0.93(0.75-1.15)$ & $0.87(0.70-1.09)$ & $0.71(0.57-0.88)$ & $0.82(0.67-1.02)$ & \\
\hline Model 3 & 1 & $0.94(0.76-1.16)$ & $0.89(0.71-1.11)$ & $0.72(0.58-0.90)$ & $0.84(0.68-1.04)$ & 0.02 \\
\hline \multicolumn{7}{|c|}{ High blood pressure ${ }^{a}$} \\
\hline No. of case (\%) & $228(12.3)$ & $216(11.6)$ & $216(10.9)$ & $205(11.4)$ & $263(14.4)$ & \\
\hline Model 1 & 1 & $1.02(0.79-1.31)$ & $0.92(0.72-1.18)$ & $0.81(0.63-1.04)$ & $0.83(0.65-1.07)$ & \\
\hline Model 2 & 1 & $1.05(0.82-1.35)$ & $0.96(0.75-1.23)$ & $0.85(0.66-1.09)$ & $0.89(0.69-1.14)$ & \\
\hline Model 3 & 1 & $1.07(0.83-1.37)$ & $0.98(0.77-1.25)$ & $0.86(0.67-1.10)$ & $0.91(0.71-1.17)$ & 0.36 \\
\hline \multicolumn{7}{|c|}{ Fasting hyperglycemia ${ }^{a}$} \\
\hline No. of case (\%) & $248(13.4)$ & $254(13.6)$ & $251(12.7)$ & $234(13.0)$ & $281(15.4)$ & \\
\hline Model 1 & 1 & $1.09(0.87-1.37)$ & $0.93(0.73-1.18)$ & $0.90(0.71-1.14)$ & $0.93(0.74-1.19)$ & \\
\hline Model 2 & 1 & $1.12(0.89-1.40)$ & $0.95(0.74-1.20)$ & $0.93(0.73-1.18)$ & $0.97(0.76-1.23)$ & \\
\hline Model 3 & 1 & $1.11(0.88-1.40)$ & $0.96(0.75-1.22)$ & $0.94(0.74-1.20)$ & $0.98(0.77-1.24)$ & 0.60 \\
\hline \multicolumn{7}{|c|}{ Hypertriglyceridemia $^{a}$} \\
\hline No. of case (\%) & $290(15.7)$ & $258(13.8)$ & $256(13.0)$ & $257(14.3)$ & $307(16.8)$ & \\
\hline Model 1 & 1 & $0.98(0.77-1.20)$ & $0.77(0.61-0.97)$ & $0.80(0.64-1.00)$ & $0.81(0.65-1.01)$ & \\
\hline Model 2 & 1 & $1.02(0.82-1.27)$ & $0.84(0.67-1.05)$ & $0.89(0.71-1.11)$ & $0.90(0.72-1.12)$ & \\
\hline Model 3 & 1 & $1.03(0.83-1.28)$ & $0.86(0.68-1.08)$ & $0.91(0.73-1.14)$ & $0.93(0.75-1.15)$ & 0.68 \\
\hline \multicolumn{7}{|l|}{ Low HDL-C ${ }^{\mathrm{a}}$} \\
\hline No. of case (\%) & $914(49.5)$ & $932(50.0)$ & $935(47.4)$ & $865(48.2)$ & $951(52.3)$ & \\
\hline Model 1 & 1 & $1.15(0.99-1.34)$ & $1.04(0.88-1.22)$ & $0.99(0.84-1.17)$ & $1.14(0.98-1.33)$ & \\
\hline Model 2 & 1 & $1.19(1.02-1.39)$ & $1.08(0.91-1.27)$ & $1.04(0.88-1.22)$ & $1.15(0.98-1.34)$ & \\
\hline Model 3 & 1 & $1.18(1.01-1.39)$ & $1.08(0.91-1.28)$ & $1.04(0.89-1.23)$ & $1.14(0.98-1.34)$ & 0.59 \\
\hline
\end{tabular}

All analyses accounted for the complex sampling design effect and the appropriate sampling weights of the national survey. Multivariate adjusted logistic regression was used to estimate the odds ratio ( $95 \%$ confidence interval).

HDL-C, high density lipoprotein cholesterol.

${ }^{a}$ Cardiometabolic syndrome was defined using the National Cholesterol Education Program Adult Treatment Panel III criteria with a modified waist circumference cutoff for Korean adults if any three or more of the five components were present. Five components: (1) abdominal obesity (waist circumference $\geq 90 \mathrm{~cm}$ in male, $\geq 85 \mathrm{~cm}$ in female); (2) high blood pressure ( $\geq 130 / 85 \mathrm{~mm} \mathrm{Hg}$ ); (3) fasting hyperglycemia ( $\geq 100 \mathrm{mg} / \mathrm{dL}$ ); (4) hypertriglyceridemia ( $\geq 150 \mathrm{mg} / \mathrm{dL}$ ); and (5) low HDL-C ( $<40 \mathrm{mg} / \mathrm{dL}$ in male, $<50 \mathrm{mg} / \mathrm{dL}$ in female), ${ }^{b}$ Model 1: adjusted for age (continuous), ${ }^{c}$ Model 2: same as model 1 and additionally adjusted for education (high school or less, college or more), income (quartile of equivalized household income), physical activity (low, moderate, high), smoking status (never smoker, past smoker, current smoker), and alcohol consumption (times/week), ${ }^{\mathrm{d}}$ Model 3: same as model 2 and additionally adjusted for total energy (kcal/day), carbohydrate (g/day), total fat (g/day), protein (g/day), sodium (mg/day) intakes as continuous variable, ${ }^{\mathrm{e}} \mathrm{P}$ for trend using median value of soybean product intake quintile after adjustments for confounders as same as model 3 . 
Table 4. Multivariable adjusted odds ratio for the cardiometabolic syndrome and its individual components, sensitivity analysis

\begin{tabular}{|c|c|c|c|c|c|c|}
\hline \multirow{2}{*}{ Variable } & \multicolumn{6}{|c|}{ Soybean food intake (soybean, soybean milk) } \\
\hline & Q1 & Q2 & Q3 & Q4 & Q5 & $P$ for trend \\
\hline \multicolumn{7}{|c|}{ Cardiometabolic syndrome ${ }^{a}$} \\
\hline No. of case (\%) & $242(12.78)$ & $189(10.41)$ & $203(10.77)$ & $194(10.07)$ & $256(14.49)$ & \\
\hline Model $1^{\mathrm{b}}$ & 1 & $0.69(0.53-0.90)$ & $0.68(0.53-0.87)$ & $0.53(0.42-0.68)$ & $0.70(0.55-0.90)$ & \\
\hline Model $2^{\mathrm{c}}$ & 1 & $0.74(0.56-0.96)$ & $0.74(0.58-0.95)$ & $0.59(0.46-0.76)$ & $0.77(0.60-0.99)$ & \\
\hline Model $3^{\mathrm{d}}$ & 1 & $0.75(0.57-0.98)$ & $0.76(0.59-0.98)$ & $0.61(0.48-0.79)$ & $0.80(0.62-1.02)$ & 0.00 \\
\hline \multicolumn{7}{|c|}{ Abdominal obesity ${ }^{a}$} \\
\hline No. of case (\%) & $367(19.38)$ & $298(16.41)$ & $302(16.02)$ & $297(15.41)$ & $358(20.26)$ & \\
\hline Model 1 & 1 & $0.77(0.63-0.95)$ & $0.70(0.57-0.87)$ & $0.61(0.50-0.75)$ & $0.70(0.57-0.86)$ & \\
\hline Model 2 & 1 & $0.82(0.67-1.02)$ & $0.76(0.61-0.94)$ & $0.67(0.54-0.82)$ & $0.76(0.61-0.94)$ & \\
\hline Model 3 & 1 & $0.83(0.67-1.03)$ & $0.77(0.62-0.95)$ & $0.68(0.56-0.84)$ & $0.78(0.63-0.97)$ & 0.04 \\
\hline \multicolumn{7}{|c|}{ High blood pressure $^{a}$} \\
\hline No. of case (\%) & $224(11.83)$ & $200(11.01)$ & $215(11.41)$ & $216(11.21)$ & $273(15.45)$ & \\
\hline Model 1 & 1 & $0.84(0.65-1.08)$ & $0.78(0.61-0.99)$ & $0.70(0.54-0.89)$ & $0.81(0.62-1.05)$ & \\
\hline Model 2 & 1 & $0.86(0.67-1.11)$ & $0.80(0.63-1.01)$ & $0.73(0.57-0.94)$ & $0.85(0.66-1.10)$ & \\
\hline Model 3 & 1 & $0.88(0.68-1.14)$ & $0.81(0.64-1.03)$ & $0.74(0.58-0.96)$ & $0.88(0.68-1.14)$ & 0.24 \\
\hline \multicolumn{7}{|c|}{ Fasting hyperglycemia $^{a}$} \\
\hline No. of case (\%) & $253(13.36)$ & $244(13.44)$ & $237(12.57)$ & $253(13.13)$ & $282(15.96)$ & \\
\hline Model 1 & 1 & $1.02(0.81-1.28)$ & $0.81(0.65-1.02)$ & $0.81(0.65-1.02)$ & $0.89(0.71-1.13)$ & \\
\hline Model 2 & 1 & $1.04(0.82-1.32)$ & $0.83(0.66-1.04)$ & $0.85(0.67-1.06)$ & $0.92(0.73-1.18)$ & \\
\hline Model 3 & 1 & $1.05(0.83-1.33)$ & $0.84(0.67-1.07)$ & $0.87(0.69-1.09)$ & $0.94(0.73-1.19)$ & 0.29 \\
\hline \multicolumn{7}{|c|}{ Hypertriglyceridemia $^{a}$} \\
\hline No. of case (\%) & $296(15.63)$ & $252(13.88)$ & $241(12.79)$ & $280(14.53)$ & $300(16.98)$ & \\
\hline Model 1 & 1 & $0.83(0.66-1.03)$ & $0.68(0.54-0.85)$ & $0.72(0.58-0.90)$ & $0.75(0.60-0.94)$ & \\
\hline Model 2 & 1 & $0.88(0.71-1.11)$ & $0.74(0.59-0.93)$ & $0.80(0.64-1.00)$ & $0.83(0.66-1.04)$ & \\
\hline Model 3 & 1 & $0.90(0.72-1.13)$ & $0.76(0.61-0.96)$ & $0.82(0.66-1.03)$ & $0.86(0.69-1.08)$ & 0.56 \\
\hline \multicolumn{7}{|l|}{ Low HDL-C ${ }^{a}$} \\
\hline No. of case (\%) & $936(49.42)$ & $886(48.79)$ & $901(47.80)$ & $942(48.88)$ & $934(52.86)$ & \\
\hline Model 1 & 1 & $0.97(0.83-1.13)$ & $0.97(0.84-1.13)$ & $0.95(0.81-1.12)$ & $1.10(0.94-1.28)$ & \\
\hline Model 2 & 1 & $1.00(0.85-1.16)$ & $1.01(0.87-1.19)$ & $0.98(0.84-1.15)$ & $1.09(0.94-1.28)$ & \\
\hline Model 3 & 1 & $1.01(0.86-1.18)$ & $1.03(0.88-1.21)$ & $0.99(0.85-1.17)$ & $1.10(0.94-1.29)$ & 0.97 \\
\hline
\end{tabular}

All analyses accounted for the complex sampling design effect and the appropriate sampling weights of the national survey. Multivariate adjusted logistic regression was used to estimate the odds ratio ( $95 \%$ confidence interval).

HDL-C, high density lipoprotein cholesterol.

${ }^{a}$ Cardiometabolic syndrome was defined using the National Cholesterol Education Program Adult Treatment Panel III criteria with a modified waist circumference cutoff for Korean adults if any three or more of the five components were present. Five components: (1) abdominal obesity (waist circumference $\geq 90 \mathrm{~cm}$ in male, $\geq 85 \mathrm{~cm}$ in female); (2) high blood pressure ( $\geq 130 / 85 \mathrm{~mm} \mathrm{Hg}$ ); (3) fasting hyperglycemia ( $\geq 100 \mathrm{mg} / \mathrm{dL}$ ); (4) hypertriglyceridemia ( $\geq 150 \mathrm{mg} / \mathrm{dL}$ ); and (5) low HDL-C ( $<40 \mathrm{mg} / \mathrm{dL}$ in male, $<50 \mathrm{mg} / \mathrm{dL}$ in female), ${ }^{b}$ Model 1: adjusted for age (continuous), 'Model 2: same as model 1 and additionally adjusted for education (high school or less, college or more), income (quartile of equivalized household income), physical activity (low, moderate, high), smoking status (never smoker, past smoker, current smoker), and alcohol consumption (times/week), ${ }^{\mathrm{d}}$ Model 3: same as model 2 and additionally adjusted for total energy (kcal/day), carbohydrate (g/day), total fat (g/day), protein (g/day), sodium intakes (mg/day) as continuous variables, ${ }^{\mathrm{e}} \mathrm{P}$ for trend using median value of soybean product intake quintile after adjustments for confounders as same as model 3. 
nents across soybean consumption. Meanwhile, after excluding tofu items from FFQ data, lower ORs and significant associations were found for several CMS components across soybean food consumption groups than those when considering all soybean food items.

For CMS prevalence (Table 4), after adjusting for age, all quintiles had significantly lower ORs than the 1st quintile in model 1. After adjusting for age, education, income, smoking status, and alcohol consumption in model 2, the significance was maintained across quintiles. After additionally adjusting for total energy intake, carbohydrate intake, fat intake, protein intake, and sodium intake in model 3 , the 2 nd ( 2 or more than 2 to 4 or less than 4 times/week), 3rd (more than 4 to 8.5 or more than 8.5 times/week), and 4 th quintile of soybean intake also had significantly lower ORs than the lowest quintile ([OR, 0.75; $95 \% \mathrm{CI}, 0.57$ to 0.98 ], [OR, 0.76 ; $95 \% \mathrm{CI}, 0.59$ to 0.98 ], and [OR, 0.61; 95\% CI, 0.48 to 0.79 ], respectively). However, the 5 th quintile of soybean intake had $20 \%$ lower OR than the reference group with $95 \%$ CI of 0.62 to 1.02 which was marginally significant. ORs for CMS prevalence significantly decreased by the quintile of soybean food intake in model 3 ( $P$ for trend $<0.00$ ).

For AO prevalence after adjusting for age (Table 4), all quintiles of soybean food intake had significantly lower ORs than the reference group. After additionally adjusting for education, income, smoking status, and alcohol consumption in model 2, the significance was maintained except for OR of the 2 nd quintile of soybean food intake. After additionally adjusting for total energy and four nutrients' intake in model 3, the 3rd, 4th, and 5 th quintiles of soybean intake showed significantly lower ORs than the 1 st quintile ([OR, $0.77 ; 95 \% \mathrm{CI}, 0.62$ to 0.95$]$, [OR, 0.68 ; $95 \% \mathrm{CI}, 0.56$ to 0.84 ], and [OR, 0.78 ; $95 \% \mathrm{CI}, 0.63$ to

Table 5. Multivariable adjusted odds ratio for the cardiometabolic syndrome score

\begin{tabular}{|c|c|c|c|c|c|}
\hline \multirow{2}{*}{ Variable } & \multicolumn{5}{|c|}{ Soybean food intake } \\
\hline & Q1 & Q2 & Q3 & Q4 & Q5 \\
\hline \multicolumn{6}{|l|}{ CMS score $=3^{\mathrm{a}}$} \\
\hline No. of case (\%) & $167(9.05)$ & $144(7.73)$ & $137(6.95)$ & $134(7.46)$ & $191(10.50)$ \\
\hline Model $1^{\mathrm{b}}$ & 1 & $0.81(0.60-1.09)$ & $0.71(0.53-0.95)$ & $0.66(0.49-0.88)$ & $0.84(0.64-1.10)$ \\
\hline Model $2^{c}$ & 1 & $0.86(0.64-1.15)$ & $0.76(0.57-1.02)$ & $0.72(0.54-0.97)$ & $0.90(0.69-1.19)$ \\
\hline Model $3^{\mathrm{d}}$ & 1 & $0.87(0.64-1.17)$ & $0.78(0.58-1.06)$ & $0.74(0.55-0.99)$ & $0.94(0.71-1.23)$ \\
\hline \multicolumn{6}{|l|}{ CMS score $=4^{\mathrm{a}}$} \\
\hline No. of case (\%) & $53(2.87)$ & $49(2.63)$ & $59(2.99)$ & $41(2.28)$ & $55(3.02)$ \\
\hline Model 1 & 1 & $1.07(0.64-1.79)$ & $1.19(0.75-1.90)$ & $0.69(0.41-1.16)$ & $0.74(0.46-1.19)$ \\
\hline Model 2 & 1 & $1.14(0.68-1.92)$ & $1.34(0.85-2.10)$ & $0.81(0.48-1.37)$ & $0.86(0.53-1.38)$ \\
\hline Model 3 & 1 & $1.13(0.68-1.89)$ & $1.36(0.86-2.13)$ & $0.83(0.49-1.42)$ & $0.86(0.53-1.40)$ \\
\hline \multicolumn{6}{|l|}{ CMS score $=5^{\mathrm{a}}$} \\
\hline No. of case (\%) & $16(0.87)$ & $12(0.64)$ & $9(0.45)$ & $7(0.39)$ & $9(0.49)$ \\
\hline Model 1 & 1 & $1.01(0.44-2.33)$ & $0.64(0.24-1.68)$ & $0.54(0.20-1.47)$ & $0.35(0.13-0.92)$ \\
\hline Model 2 & 1 & $1.03(0.45-2.37)$ & $0.67(0.25-1.73)$ & $0.59(0.22-1.58)$ & $0.39(0.15-1.03)$ \\
\hline Model 3 & 1 & $1.10(0.48-2.51)$ & $0.74(0.28-1.97)$ & $0.64(0.23-1.78)$ & $0.47(0.18-1.21)$ \\
\hline
\end{tabular}

All analyses accounted for the complex sampling design effect and the appropriate sampling weights of the national survey. Multivariate adjusted logistic regression was used to estimate the odds ratio (95\% confidence interval).

CMS, cardiometabolic syndrome.

${ }^{a}$ CMS score: CMS was defined using the National Cholesterol Education Program Adult Treatment Panel III criteria with a modified waist circumference cutoff for Korean adults if any three or more of the five components were present. Five components: (1) abdominal obesity (waist circumference $\geq 90 \mathrm{~cm}$ in male, $\geq 85 \mathrm{~cm}$ in female); (2) high blood pressure ( $\geq 130 / 85 \mathrm{~mm} \mathrm{Hg}$ ); (3) fasting hyperglycemia ( $\geq 100 \mathrm{mg} / \mathrm{dL}$ ); (4) hypertriglyceridemia ( $\geq 150 \mathrm{mg} / \mathrm{dL}$ ); and (5) low HDL-C ( $<40 \mathrm{mg} / \mathrm{dL}$ in male, $<50 \mathrm{mg} / \mathrm{dL}$ in female). The number corresponding to the component was scored, ${ }^{b}$ Model 1: adjusted for age (continuous), ${ }^{c}$ Model 2: same as model 1 and additionally adjusted for education (high school or less, college or more), income (quartile of equivalized household income), physical activity (low, moderate, high), smoking status (never smoker, past smoker, current smoker), and alcohol consumption (times/week), ${ }^{\mathrm{d}}$ Model 3: same as model 2 and additionally adjusted for total energy (kcal/day), carbohydrate (g/day), total fat (g/day), protein (g/day), sodium intakes (mg/day) as continuous variables. 
0.97], respectively). In model 3 , ORs for AO prevalence significantly decreased across soybean food intake quintiles $(P$ for trend $<0.05$ ).

ORs of high BP were significantly lower in the 3rd and 4th quintiles of soybean food intake than the reference quintile (Table 4). However, after adjusting for confounders in models 2 and 3, only ORs in the 4th quintile of soybean food intake were significantly lower than the 1st quintile ([OR, 0.73; 95\% CI, 0.57 to 0.94 ] and [OR, $0.74 ; 95 \%$ CI, 0.58 to 0.96 ], respectively).

Participants in the 3rd quintile of soybean intake had the lowest OR for hypertriglyceridemia which was significant even after adjusting for confounders in models 2 and 3 ([OR, 0.74; $95 \% \mathrm{CI}, 0.59$ to 0.93 ] and [OR, $0.76 ; 95 \% \mathrm{CI}, 0.61$ to 0.96 ], respectively). ORs of the 4th group showed significantly lower OR that the reference after adjusting for age in model 1, although the significance attenuated after adjusting for various covariates in models 2 and 3.

\section{Soybean food intake and cardiometabolic syndrome score} People in the 4th quintile (8.5 to 17 times/week) of soybean food intake had 26\% lower ORs of having CMS score of 3 than those in the 1st quintile (less than 2 times/week) of soybean food intake after adjusting for confounders in model 3 (OR, 0.74; $95 \%$ CI, 0.55 to 0.99 ) (Table 5). There was no significant difference in ORs for having CMS scores of 4 and 5 across soybean food intake quintiles compared to the reference.

\section{DISCUSSION}

We evaluated the association between soybean food consumption and cardio metabolic diseases in Korean women based on nationally representative survey data. For overall soybean food, moderate soybean food consumption was associated with lower CMS prevalence and central obesity. Especially for central obesity, ORs had significant decreasing linearity across soybean food intake groups. The observed inverse association for CMS prevalence was attenuated in the highest quintile of soybean consumption (more than 17 times/week of soybean consumption). This reverse J shape relationship was also found for other CMS components as OR of the 5th group was higher than that of the 4th group. Even for AO prevalence which had significant inverse linear trend across soybean intake quintiles, OR of the 5th quintile did not show significance.

After excluding tofu items, CMS prevalence and central obe- sity had prominent decreasing association across soybean intake groups, while only moderate consumption was related to lower OR in high BP and hypertriglyceridemia. $P$ values for trend in CMS and AO prevalence using median value of soybean product intake quintile after adjusting for confounders in model 3 showed significant linearity across soybean food intake groups. Others had reverse J shape relation with soybean intake. It tended to decrease until the 4th quintile, but it increased from the fifth to the reverse.

When observing general characteristics, women in the 4th quintile of soybean product intake showed some desirable health condition-lowest BMI, highest household income, vigorous physical activity, and high energy adjusted protein intake. Meanwhile, women in the 5th quintile showed some undesirable heath condition-eldest, highest BMI, highest total energy intake, and high energy adjusted carbohydrate intake. We did some additional analysis to see the effect of each variable which was higher in the 5th group.

To determine the age modified on CMS, first we examined the interaction of soybean intake quintiles with age. However, there was no significant interaction over all quintiles for CMS components (results not shown). Secondly, as the elder women tended to be postmenopausal, we stratified participants by post- or pre-menopause. All the significance disappeared in premenopausal group. Postmenopausal women showed significantly lower ORs in the 4th and 5th quintiles of soybean intake for AO. The influence of age and menopause to the relation between soybean intake and CMS components is presumed to be small.

To determine the interaction of BMI with soybean intake, we stratified participants by BMI as obesity, pre-obesity, and under pre-obesity [32] and then analyzed CMS components. There were no significant ORs in groups that were not obesity after adjusting for various covariates in model 3 . In the obesity group, the 4th and the 5th quintiles had significantly lower ORs than the reference group for $\mathrm{AO}$ prevalence in model 3. This suggested that the lowering significance in the 5th quintile was not due to higher BMI.

Total energy intake and energy adjusted carbohydrate intake might be the reason for the reverse J shape association of CMS components with soybean intake quintiles. Park et al. [33] has reported that MetS group has higher carbohydrate intake and lower protein and fat (monounsaturated, polyunsaturated, and saturated fatty acids) intake than the non-MetS group. Carbohydrate intake was positively associated with the risk of MetS 
in a previous study [33] while protein and fat intake exhibited a prominently reverse association with the prevalence of MetS [33]. According to the study of dietary characteristics of nutrient intake with health status among Koreans, the proportion of energy intake from carbohydrates was significantly higher in the group with CMS than that in the group without CMS [34]. Results of the present study suggested that the group that consumed soybean the most frequently have eaten the highest energy adjusted carbohydrate to show inverse association with the risk of CMS. Also, effects of carbohydrate intake were different by BMI in previous research. In a cohort study, results showed a positive relation between high intake of added sugars from liquid and components of the CMS among overweight participants, but not among normal-weight participants [35]. As participants of the top quintile group of soybean food intake had the highest BMI, they might be more vulnerable to metabolic disorder than any other groups when they consumed carbohydrates.

Soy protein have been found to have implications for insulin/glucagon ratio. Isoflavones appear to influence lipid metabolism by altering gene expression for lipid-related genes [36]. By combining mRNA and macroarray analysis, genes involved in lipid metabolism, regulation of transcription and translation, protease inhibition, apoptosis, and cell proliferation regulation have been found to be expressed at higher levels in rat livers fed with low or high isoflavone soy protein diets compared to those in livers fed casein diet [37]. For human, according to a meta-analysis of 14 studies conducted on 11 cohorts, the group with the highest legume consumption was associated with a decreased risk of $10 \%$ in both cardiovascular disease and coronary heart disease compared to the group with the lowest legume consumption [38]. Recent studies in Korean adult women have shown that they mainly have intake of isoflavones from soybean foods known to have beneficial effects on several metabolic disorders [22,39,40]. Consistent with our study, previous researches have indicated that frequent consumption of soybean diet could regulate overall levels of lipid and fat accumulation in the liver which causes insulin resistance and dyslipidemia [29,41]. Some experimental studies have reported that soybean have proteins that are more effective than animal proteins in weight reduction and arterial stiffness. In addition, soybean contains isoflavonoids and saponins that can improve lipid profiles and risk of CMS $[13,42,43]$. Potential mechanisms by which soy protein and isoflavones might prevent CMS include a beneficial effect on plasma lipid concentrations, antioxidant effects, antiproliferative and antimigratory effects on smooth muscle cells, effects on thrombus formation, and maintenance of normal vascular reactivity [36].

AST to ALT ratio showed different phase in the most frequent soybean consumption group. Serum AST-to-ALT ratio is a substitute measure for NAFLD. It has been shown to be inversely associated with metabolic disorder and insulin resistance in previous clinical and epidemiological studies [44,45]. A cohort study showed that an increased AST-to-ALT ratio was correlated with a consistent reduction in the onset of CMS and its components [46]. This implies that, the more we eat soy bean food, the more beneficial it will be for our liver metabolism. At last the onset of CMS would be prolonged [29]. Since we could not observe an inverse relation of soybean food intake with insulin resistance, HbAlc level, or other probes related to glucose metabolism, it suggests that our participants might be a comparatively healthy population who are not diagnosed with T2DM, hyperlipidemia, or hypertension before analysis. Thus, we could not see any statistical significance. The beneficial effect of soybean could be shown more prominent among unhealthy people. An epidemiologic study has shown that higher intake of soy food is related to lower incidence of T2DM among overweight women [10]. Some cross-sectional studies have found that the association of soybean intake with $\mathrm{AO}$ and cholesterol level appears to be stronger among postmenopausal women than that among premenopausal women $[10,20]$.

ORs of CMS individual components according to overall soybean food intake frequency had different results compared to soybean food intake without tofu items which showed more significant decrease in AO, BP, and TG. Similarly, some observational studies have found that eating soybean food has significant negative relation with CVD, although eating tofu and miso has borderline significant association [38]. Epidemiologic and experimental studies have also reported that components in soybean are different depending on processing method which might play a role in the positive association $[47,48]$.

We acknowledge that the present study has several limitations. First, we included only women in this study. It is possible that men differ from women in effects of soybean food consumption on CMS. The reason why we did not include men was because soy consumption in Korea was especially high among women. This is because soy isoflavones are associated with female hormones. Thus, soy consumption seems to be more related to women's health. Second, our study had a cross- 
sectional design. Thus, we could not determine a causal relationship of soybean consumption with risk of CMS among Korean women. Lastly, we could not rule out the presence of unknown confounders. Some unmeasured and residual confounding factors could exist. However, it is doubtful that those confounding would completely erase the association found in the present study. In addition, the assessment of soybean intake was mainly based on questions related to the intake of food ingredients-soybean milk, tofu, and soybean. We were unable to distinguish the effect of different processing types of soybean food (fermented, fried, unsweetened food, etc.). However, other studies on Korean adult women have shown that ingestion of soy isoflavones is mainly induced by intake of soybean paste, soybean milk, and tofu, although there is a difference in the level of soybean food intake [20,39]. So identifying the frequency of eating soybean foods in Korean women may have a similar effect as comparing the nutrient intake of soybeans. Although this study had limitations, we adjusted for several potential confounders in our analyses.

The present study also has strengths. KNHANES had a large nationally representative sampling design that provided detailed information, thus allowing for better control of potential confounders among the Korean population. These results could provide insight into the influence of soy consumption on general population statistically. In addition, we conducted a sensitivity analysis to determine the association varied from each soybean food item. When we analyzed the association with total soybean food item, the result showed that only AO had significant association. In Asia, there are plenty of processing method using soy bean. Thus, it was unclear whether each subgroup of soybean food would show the same result with CMS prevalence.

In conclusion, we found that moderate consumption of soybean food showed negative association with having CMS and AO among healthy women. Our results warrant further studies such as randomized controlled trials about the effect of various kinds of processed soybean food intake frequency on CMS prevalence. If our results are confirmed by further research, it could encourage the consumption of soybean food as a healthy alternative to Western-style meals.

\section{CONFLICTS OF INTEREST}

No potential conflict of interest relevant to this article was reported.

\section{AUTHOR CONTRIBUTIONS}

Conception or design: W.K.S., Y.K.

Acquisition, analysis, or interpretation of data: S.H.J., W.K.S.

Drafting the work or revising: S.H.J., W.K.S., Y.K.

Final approval of the manuscript: Y.K.

\section{ORCID}

Sook-Hyun Jun https://orcid.org/0000-0002-3186-4640

Woo-Kyoung Shin https://orcid.org/0000-0003-2725-4652

Yookyung Kim https://orcid.org/0000-0002-8438-0121

\section{ACKNOWLEDGMENTS}

This research was supported by the College of Education, Korea University Grant in 2019 and conducted using raw data from the KNHANES (2007 to 2011) performed by the Korean Centers for Disease Control and Prevention.

\section{REFERENCES}

1. World Health Organization: Noncommunicable diseases country profiles 2011. Available from: http://www.who.int/ nmh/publications/ncd_profiles2011/en/(cited 2019 Oct 22).

2. Sapbamrer R, Visavarungroj N, Suttajit M. Effects of dietary traditional fermented soybean on reproductive hormones, lipids, and glucose among postmenopausal women in northern Thailand. Asia Pac J Clin Nutr 2013;22:222-8.

3. Tran BT, Jeong BY, Oh JK. The prevalence trend of metabolic syndrome and its components and risk factors in Korean adults: results from the Korean National Health and Nutrition Examination Survey 2008-2013. BMC Public Health 2017;17: 71.

4. Aguilar M, Bhuket T, Torres S, Liu B, Wong RJ. Prevalence of the metabolic syndrome in the United States, 2003-2012. JAMA 2015;313:1973-4.

5. Tovar J, Johansson M, Bjorck I. A multifunctional diet improves cardiometabolic-related biomarkers independently of weight changes: an 8-week randomized controlled intervention in healthy overweight and obese subjects. Eur J Nutr 2016;55:2295-306.

6. Ministry of Health and Welfare (South Korea). '95 National Nutrition Survey Report, No 40000-65312-56-04. Seoul: Ministry of Health and Welfare; 1997. p.115-70. 
7. Van Erp-Baart MA, Brants HA, Kiely M, Mulligan A, Turrini A, Sermoneta C, Kilkkinen A, Valsta LM. Isoflavone intake in four different European countries: the VENUS approach. Br J Nutr 2003;89 Suppl 1:S25-30.

8. Chun OK, Chung SJ, Song WO. Urinary isoflavones and their metabolites validate the dietary isoflavone intakes in US adults. J Am Diet Assoc 2009;109:245-54.

9. Messina MJ. Legumes and soybeans: overview of their nutritional profiles and health effects. Am J Clin Nutr 1999;70(3 Suppl):439S-50S.

10. Nanri A, Mizoue T, Takahashi Y, Kirii K, Inoue M, Noda M, Tsugane S. Soy product and isoflavone intakes are associated with a lower risk of type 2 diabetes in overweight Japanese women. J Nutr 2010;140:580-6.

11. Aoyama T, Fukui K, Nakamori T, Hashimoto Y, Yamamoto T, Takamatsu K, Sugano M. Effect of soy and milk whey protein isolates and their hydrolysates on weight reduction in genetically obese mice. Biosci Biotechnol Biochem 2000;64:2594600 .

12. Bhathena SJ, Velasquez MT. Beneficial role of dietary phytoestrogens in obesity and diabetes. Am J Clin Nutr 2002;76:1191201.

13. Cai Y, Guo K, Chen C, Wang P, Zhang B, Zhou Q, Mei F, Su Y. Soya isoflavone consumption in relation to carotid intima-media thickness in Chinese equol excretors aged 40-65 years. Br J Nutr 2012;108:1698-704.

14. Rosell MS, Appleby PN, Spencer EA, Key TJ. Soy intake and blood cholesterol concentrations: a cross-sectional study of 1033 pre- and postmenopausal women in the Oxford arm of the European Prospective Investigation into Cancer and Nutrition. Am J Clin Nutr 2004;80:1391-6.

15. Morimoto Y, Steinbrecher A, Kolonel LN, Maskarinec G. Soy consumption is not protective against diabetes in Hawaii: the Multiethnic Cohort. Eur J Clin Nutr 2011;65:279-82.

16. Matthan NR, Jalbert SM, Ausman LM, Kuvin JT, Karas RH, Lichtenstein AH. Effect of soy protein from differently processed products on cardiovascular disease risk factors and vascular endothelial function in hypercholesterolemic subjects. Am J Clin Nutr 2007;85:960-6.

17. Saraf-Bank S, Esmaillzadeh A, Faghihimani E, Azadbakht L. Effects of legume-enriched diet on cardiometabolic risk factors among individuals at risk for diabetes: a crossover study. J Am Coll Nutr 2016;35:31-40.

18. Pan A, Franco OH, Ye J, Demark-Wahnefried W, Ye X, Yu Z, Li $\mathrm{H}$, Lin X. Soy protein intake has sex-specific effects on the risk of metabolic syndrome in middle-aged and elderly Chinese. J Nutr 2008;138:2413-21.

19. Jenkins DJ, Kendall CW, Connelly PW, Jackson CJ, Parker T, Faulkner D, Vidgen E. Effects of high- and low-isoflavone (phytoestrogen) soy foods on inflammatory biomarkers and proinflammatory cytokines in middle-aged men and women. Metabolism 2002;51:919-24.

20. Lee MJ, Sohn CY, Park OJ. Relation between health status and intake of soy isoflavone among adult women in Seoul. J East Asian Soc Diet Life 2010;20:218-30.

21. Ye YB, Chen AL, Lu W, Zhuo SY, Liu J, Guan JH, Deng WP, Fang S, Li YB, Chen YM. Daidzein and genistein fail to improve glycemic control and insulin sensitivity in Chinese women with impaired glucose regulation: a double-blind, randomized, placebo-controlled trial. Mol Nutr Food Res 2015;59: 240-9.

22. Byun MS, Yu OK, Cha YS, Park TS. Korean traditional Chungkookjang improves body composition, lipid profiles and atherogenic indices in overweight/obese subjects: a double-blind, randomized, crossover, placebo-controlled clinical trial. Eur J Clin Nutr 2016;70:1116-22.

23. Kwon DY, Daily JW 3rd, Kim HJ, Park S. Antidiabetic effects of fermented soybean products on type 2 diabetes. Nutr Res 2010; 30:1-13.

24. Ministry of Health and Welfare: Welfare and family welfare dietary reference intakes for Koreans 2015. Available from: https://www.mohw.go.kr (cited 2019 Oct 22).

25. Koo S, Kim Y, Kim MK, Yoon JS, Park K. Nutrient intake, lifestyle factors and prevalent hypertension in Korean adults: results from 2007-2008 Korean National Health and Nutrition Examination Survey. Korean J Community Nutr 2012;17:32940.

26. Kang K, Sung J, Kim CY. High risk groups in health behavior defined by clustering of smoking, alcohol, and exercise habits: National Heath and Nutrition Examination Survey. J Prev Med Public Health 2010;43:73-83.

27. Lee SY, Park HS, Kim DJ, Han JH, Kim SM, Cho GJ, Kim DY, Kwon HS, Kim SR, Lee CB, Oh SJ, Park CY, Yoo HJ. Appropriate waist circumference cutoff points for central obesity in Korean adults. Diabetes Res Clin Pract 2007;75:72-80.

28. Matthews DR, Hosker JP, Rudenski AS, Naylor BA, Treacher DF, Turner RC. Homeostasis model assessment: insulin resistance and beta-cell function from fasting plasma glucose and insulin concentrations in man. Diabetologia 1985;28:412-9.

29. Lim S, Oh TJ, Koh KK. Mechanistic link between nonalcoholic 
fatty liver disease and cardiometabolic disorders. Int J Cardiol 2015;201:408-14.

30. Angulo P. Nonalcoholic fatty liver disease. N Engl J Med 2002; 346:1221-31.

31. Kotronen A, Peltonen M, Hakkarainen A, Sevastianova K, Bergholm R, Johansson LM, Lundbom N, Rissanen A, Ridderstrale M, Groop L, Orho-Melander M, Yki-Jarvinen H. Prediction of non-alcoholic fatty liver disease and liver fat using metabolic and genetic factors. Gastroenterology 2009;137:865-72.

32. Treatment Guidelines Commission of the Korean Society for the Study of Obesity. Obesity treatment guidelines 2018. Seoul: Cheongun; 2018.

33. Park S, Ahn J, Kim NS, Lee BK. High carbohydrate diets are positively associated with the risk of metabolic syndrome irrespective to fatty acid composition in women: the KNHANES 2007-2014. Int J Food Sci Nutr 2017;68:479-87.

34. Jung HJ, Song WO, Paik HY, Joung H. Dietary characteristics of macronutrient intake and the status of metabolic syndrome among Koreans. Korean J Nutr 2011;44:119-30.

35. Wang JW, Mark S, Henderson M, O'Loughlin J, Tremblay A, Wortman J, Paradis G, Gray-Donald K. Adiposity and glucose intolerance exacerbate components of metabolic syndrome in children consuming sugar-sweetened beverages: QUALITY cohort study. Pediatr Obes 2013;8:284-93.

36. Torres N, Torre-Villalvazo I, Tovar AR. Regulation of lipid metabolism by soy protein and its implication in diseases mediated by lipid disorders. J Nutr Biochem 2006;17:365-73.

37. Iqbal MJ, Yaegashi S, Ahsan R, Lightfoot DA, Banz WJ. Differentially abundant mRNAs in rat liver in response to diets containing soy protein isolate. Physiol Genomics 2002;11:219-26.

38. Yan Z, Zhang X, Li C, Jiao S, Dong W. Association between consumption of soy and risk of cardiovascular disease: a metaanalysis of observational studies. Eur J Prev Cardiol 2017;24: 735-47.
39. Lee MJ, Kim JH. Estimated dietary isoflavone intake among Korean adults. Nutr Res Pract 2007;1:206-11.

40. Choi MK, Kim MH, Sung CJ, Lee WY, Park JD. A study on relation among habitual isoflavone intake, blood pressure, and serum lipid parameters in Korean men and women over 20 years old. Korean J Community Nutr 2005;10:493-500.

41. Paul G, Mendelson GJ. Evidence supports the use of soy protein to promote cardiometabolic health and muscle development. J Am Coll Nutr 2015;34 Suppl 1:56-9.

42. Van Nielen M, Feskens EJ, Rietman A, Siebelink E, Mensink M. Partly replacing meat protein with soy protein alters insulin resistance and blood lipids in postmenopausal women with abdominal obesity. J Nutr 2014;144:1423-9.

43. Anthony MS, Clarkson TB, Williams JK. Effects of soy isoflavones on atherosclerosis: potential mechanisms. Am J Clin Nutr 1998;68(6 Suppl):1390S-3S.

44. Sorbi D, Boynton J, Lindor KD. The ratio of aspartate aminotransferase to alanine aminotransferase: potential value in differentiating nonalcoholic steatohepatitis from alcoholic liver disease. Am J Gastroenterol 1999;94:1018-22.

45. Hanley AJ, Williams K, Festa A, Wagenknecht LE, D’Agostino RB Jr, Haffner SM. Liver markers and development of the metabolic syndrome: the insulin resistance atherosclerosis study. Diabetes 2005;54:3140-7.

46. Yadav D, Choi E, Ahn SV, Baik SK, Cho YZ, Koh SB, Huh JH, Chang Y, Sung KC, Kim JY. Incremental predictive value of serum AST-to-ALT ratio for incident metabolic syndrome: the ARIRANG Study. PLoS One 2016;11:e0161304.

47. Mueller NT, Odegaard AO, Gross MD, Koh WP, Yu MC, Yuan JM, Pereira MA. Soy intake and risk of type 2 diabetes in Chinese Singaporeans [corrected]. Eur J Nutr 2012;51:1033-40.

48. Joo EY, Park CS. Antioxidant and fibrinolytic activities of extracts from soybean and Chungkukjang (fermented soybean paste). Korean J Food Preserv 2011;18:930-7. 